Lesungen (Décsy, Gyula, Der Münchener Kodex II. Das ungarische Hussiten-Evangeliar aus dem 15. Jahrhundert, Buchstabentreuer Abdruck, Otto Harrassowitz, Wiesbaden 1966; Nyiri, Antal [red.], A Müncheni Kodex 1466-ból [Der Münchener Kodex aus dem Jahre 1466], Akadémiai Kiadó, Budapest 1971) nachzuschlagen.

Dessenungeachtet stellt Adám Szabó mit diesem Werk der Fennougristik Primärmaterial zur Verfügung, was gleichermassen für Sprachwissenschaftler, Stilisten, Literaturwissenschaftler und Ethnographen als wertvolle Ausgangsbasis dienen kann.

RUDOLF BARTSCH

Eine Neuerscheinung aus dem Bereich des Wogulischen

BÉla Kálmán, Wogulische Texte mit einem Glossar. Akadémiai Kiadó, Budapest 1976. $354 \mathrm{~S}$.

Béla Kálmán ist heute global gesehen der führende Wogulist. In dankenswerter Weise hat er durch seine Publikationen die Beschäftigung mit seinem Fach gefördert; das vorliegende Buch enthält Proben der nördlichen Dialekte von der Sygva, Sosva und vom $\mathrm{Ob}$ sowie aus dem zur östlichen Dialektgruppe gehörenden Jukonda-Dialekt. Durch diese Texte werden u.a. die umfangreichen Sammlungen von Artturi Kannisto insofern ergänzt, als die genannten Dialekte mit Ausnahme der Mundart von der Sosva bei Kannisto fehlen. Allerdings sind die Unterschiede zwischen den nördlichen Dialekten minimal: es handelt sich dabei in der Hauptsache um einige einfache lautliche Besonderheiten, die vom Standpunkt des Systems bedeutungslos sind. Ferner kommt der Einfluss des Ostjakischen im Lautbestand und in der Lexik des Dialekts vom Ob besonders deutlich zum Ausdruck, was sich natürlich durch die langanhaltenden engen nachbarlichen Kontakte erklärt.

Zusätzlich zu den Texten enthält das Buch einen komprimierten grammatischen Teil. In der Lautlehre kommt Kálmán wieder einmal auf das Problem der Quantitätskorrelation im Wogulischen zurück. Wolfgang Steinitz hat schon früher die von Artturi Kannisto aufgezeichneten komplizierten Längen als binäre Opposition reduziert, mit welcher Lösung sich auch Kálmán begnügt, allerdings mit Bedenken, da er selbst diese Opposition nicht habe feststellen können. Vor allem in den 
Fällen, wo auch nicht der geringste qualitative Unterschied die Quantitätsdistinktion unterstützte, entstanden laufend Fehldeutungen. Dasselbe Problem schien auch die jungen Wogulen selbst zu stören. Als ich im Herbst 1971 im selben Herzen-Institut in Leningrad, wo auch Kálmán seine Untersuchungen durchgeführt hat, einige wogulische Studenten interviewte, stellte ich bei der Verwendung von Minimalpaar-Tests fest, dass die Quantitätsopposition zumindest im Sprachgefühl dieser Informanten geschwunden war. Als sie merkten, worum es in dem Test ging, bekräftigten sie dies noch durch die Aussage, es bestehe kein Unterschied. Man sollte nicht vergessen, dass dieser Unterschied im heute bei den Wogulen üblichen kyrillischen Schriftsystem nicht bezeichnet wird, was einen Anteil daran haben kann, dass der frühere phonologische Gegensatz geschwunden oder in einigen Fällen zu einer qualitativen Opposition geworden ist. Das Obenstehende gilt nur für die nördlichen Dialekte; für die Mundart von Jukonda lässt sich schwer etwas Endgültiges sagen, da das Material recht spärlich ist und die Informanten von Kálmán, die bereits über zehn Jahre nicht mehr in ihrer Heimat wohnen und lieber Russisch als Wogulisch sprechen, in dieser Hinsicht keine besonders zuverlässigen Quellen sind.

Die Musterparadigmen sind aus den Dialekten von der Sygva, vom Ob und von der Jukonda ausgewählt. Die Tabellen vermitteln den Eindruck, als seien die systematischen Unterschiede zwischen den genannten Mundarten sehr klein. Die wenigen vorhandenen Lücken beruhen allgemein nur auf dem Mangel an Material, nicht darauf, dass die Flexionsformen selbst in dem betreffenden Dialekt fehlen. Kálmán weist denn auch in seinem Vorwort auf die Schwierigkeiten hin, die mit der Erstellung der Paradigmen verbunden waren. Hier besteht natürlich ausserdem immer das Problem, dass die Informanten nur die Formen nennen, nach denen der Forscher sie tatsächlich fragt. Ausserdem weiss jeder aus eigener Erfahrung, wie schwer es ist - nachdem man eine Zeitlang Wörter flektiert hat - zu entscheiden, welche Form wirklich richtig und existent ist und welche nur das Produkt eines vorübergehend unsicheren Sprachgefühls darstellt. Deshalb könnte ein zuverlässigeres Ergebnis nur durch das Exzerpieren von Texten erreicht werden; diese Arbeit aber kann erst mittels EDV-Methoden und anhand wirklich umfangreicher Materialien durchgeführt werden.

Gemäss der finnougristischen Tradition sind die Texte ins Deutsche übersetzt; am Ende steht ausserdem auch eine ungarische Übersetzung. Es ist jedoch auch mit Hilfe dieser Übersetzungen nicht leicht, den wogulischen Originaltext zu 
analysieren, denn Wortfolge, syntaktische Konstruktionen und Denkweise der Sprache sind im Deutschen und in den finnisch-ugrischen Sprachen sehr unterschiedlich. Da die deutsche Übersetzung auch noch bedeutend länger ist als das Original, ist es mitunter sogar schwer, die einander entsprechenden Stellen zu finden, vor allem in den Erzählungen, wo sich die Geschehnisse fast gleichartig häufen. Die Numerierung der Sätze oder wenigstens der Absätze hätte hier einiges erleichtert. Mit Hilfe der Musterparadigmen und des Wortschatzes kann der Leser dagegen wenigstens in den meisten Fällen die syntaktische Konstruktion klären. Es ist jedoch sicher, dass derjenige, der lediglich mit Hilfe dieses Buches das Wogulische erlernt, für die kompliziertesten Fragen dringend Morphem-Übersetzungen oder Kommentare brauchte. Die Bedeutung eines Ausdrucks zu kennen und die Konstruktion zu verstehen, ist ja durchaus nicht ein und dasselbe, zumindest nicht in der Linguistik.

Durch Interviewen seiner Informanten hat Kálmán auch wogulische Lieder aufgezeichnet, deren Text und Melodie am Schlusse des Buches mitgeteilt werden.

KAISA HÄKKINEN

\section{Material in Sodankylä Lappish}

PekKa Sammallahti, Sodankylän saamelaisten entistä elämää Elsa-Marja Aikion kertomana (The traditional way of life of Sodankylä Lapps as told by Elsa-Marja Aikio). Castrenianumin toimitteita 14. Helsinki 1975. 211 p.

The southernmost Lapps in Finland live in Vuotso, a village in the parish of Sodankylä (Lappish: Soadekilli). Originally the ancestors of these Lapps migrated from Enontekiö (Enudak) in the north, but the old Lappish population of Sodankylä died out long ago.

In 1975 Pekka Sammallahti published text-material which he had tape-recorded with Mrs. Elsa-Marja Aikio (born 1891) in Vuotso. These texts include stories (Lappish muihtalus, Finnish muistelus) about many different aspects of everyday Lappish life in earlier times. The most interesting texts are those about the reindeer, its diseases, how to prepare different kinds of meals with reindeer-meat, and so on. The chapter about the clothing of the Sodankylä Lapps is informative, too. The texts are parallel in Lappish and Finnish, with the original Lappish text on the right-hand page and the translation into Finnish on the left. 